\title{
Stress field in the Antarctic tectonic plate: elastic and plastic models
}

\author{
P. Haderka ${ }^{1}$, A. N. Galybin ${ }^{1} \&$ Sh. A. Mukhamediev ${ }^{2}$ \\ ${ }^{1}$ Wessex Institute of Technology, Southampton, UK \\ ${ }^{2}$ Institute of Physics of the Earth, Moscow, Russia
}

\begin{abstract}
The purpose of this study is to compare different rheological models of tectonic plates for the same set of input data. First, the 2D elastic tectonic stress field in Antarctic plate is modelled by employing experimental data on principal stress orientations. Then the determination of the $2 \mathrm{D}$ stress field by assuming the Antarctic lithospheric plate to be in fully plastic state follows. Boundary conditions for the plastic model are specified from the elastic solution. A finitedifference type approach based of alternation of the Cauchy boundary value problems for the construction of the slip lines and the principal stress trajectories is applied to obtain distribution of the mean stress within Antarctic plate. The significance of rheology is underpinned by comparing the elastic and plastic fields of the mean stress.
\end{abstract}

Keywords: stress field, elasticity, plasticity, geodynamics, tectonics.

\section{Introduction}

Antarctica is a key element in Earth's geodynamic and climatic systems for a variety of reasons:

- Antarctica continues to be tectonically active, Torsvik et al. [1].

- Antarctica is significant in the global relative plate motion circuit and the quantification of internal deformation of Antarctica is an essential part of any global relative-plate-motion model, [2,3].

- Antarctica plays a central role in shaping the present global environment.

Despite this importance the first-order characteristics of the lithosphere remain poorly described over large portions of the continent. 
To the best of our knowledge, no papers devoted to mathematical modelling of the regional tectonic stress field caused by driving forces applied at the Antarctic plate (AP) boundaries have been published. This can be explained by the absence of data on principal stress orientations within the plate, that is vital for obtaining the plate-scale solutions by conventional methods as it has been done for several regions, among others, for the Indo-Australian, African, West European, and North American plates [4-8]. The cited papers employ the traditional approach of the 2D elasticity and make use of experimentally obtained data on orientations of the principal stresses (mainly from World Stress Map Project database, Reinecker et al [9]) as constraints imposed on the solutions. The stress fields are calculated by solving a number of boundary value problems of elasticity often posed in terms of stresses. This presumes that the boundary stresses are chosen either hypothetically or from model considerations such that the calculated orientations of the principal stresses fit the observed stress orientations. Such an approach always leads to crucial uncertainness in the stress tensor determination because similar stress orientations can be obtained for different magnitudes of boundary stresses.

In the present study this peculiarity is overcome by the direct use of the data available without any assumptions on stress magnitudes. Analysis of the elastic constitutive equations then enables to recover the fields of mean stress and maximum shear stress. Boundary values of the former then serve as one of the boundary conditions $(\mathrm{BC})$ for the plastic problem.

Solution of the 2D ideal plastic problem where the boundary values are propagated along slip lines is well known and available in works of Hill [10] or Kachanov [11]. The solution of the Cauchy problem however is constrained inside a triangular region the legs of which are the characteristics of the $2 \mathrm{D}$ problem's equations. The main target now becomes to propagate the solution beyond the boundaries of the initial triangle in order to extend the solution surface. The concept of stress trajectories is employed for this purpose as will be shown in the text.

Important aspects of our study are as follows:

- The use of a special numerical procedure which recasts the WSMP experimental data in the form of $\mathrm{BC}$ formulated in terms of principal stress orientations specified on contour (with regard to AP).

- Development of the method of alternation of the principal stress trajectories grid and the slip lines grid while integrating the plasticity equations.

\section{Mathematical models in elasticity and plasticity}

\subsection{Data and assumptions used in modelling}

The data of A-C quality on orientations of principal stresses from the 2005 release of the WSMP database (in [9]) are used. Due to low seismic activity of AP almost all data are concentrated on the AP margins suggesting that inclination, $\varphi$, of the maximum horizontal compression within AP has a limiting values $\varphi^{ \pm}$on the contour $\Gamma$ associated with the AP boundary and these are the 
only boundary data available from observations. Other $\mathrm{BC}$ are needed for proper formulation of an elastic boundary value problem, and these can assume continuity of the stress vector across the boundary, see Galybin [12] for detail.

Basal shear stresses acting beneath the lithosphere are neglected, which can be justified by the fact that AP has a low absolute velocity with respect to the deeper mantle, as introduced in [13-14]. Therefore a 2D model reflecting driving forces applied at the AP boundary can be considered separately, while the complete 3D stress tensor can be obtained by superimposing the stresses caused by the rock weight and ice sheet load on the continental part of the plate. Gravitational potential energy differences which produce lateral body forces and have been recognised as an important source for intraplate stresses in AP, see Kreemer and Holt [15], in the elastic problem affect neither principal stress orientations nor deviatoric stresses if potential of body forces is approximated by harmonic function, as explained in Mukhamediev et al [16]. Lateral body forces caused by harmonic potential only influence the magnitudes of principal stresses by adding to them the same amount which can be different from point to point.

For simplicity it is assumed that the region (Antarctic tectonic plate) is planar, that is, Earth's curvature is neglected and the plate is projected on the equatorial plane (plane stress conditions may be adopted). It has also been assumed that the adjacent regions are isotropic.

Body forces will be absent in the computations, however they are accounted for in the BC (data on stress orientations). For the plastic solution it is assumed that the entire domain is in the state of limiting equilibrium. The model of ideal plasticity is adopted, however, this simplification is not vital and can be extended to take friction and cohesion into account.

\subsection{Mathematical model in elasticity}

The mathematical model for elastic rheology has been introduced in detail e.g. in Galybin and Mukhamediev [17]. The differential equations of equilibrium (DEE) in complex form are used further on:

$$
\frac{\partial P(z, \bar{z})}{\partial z}=\frac{\partial D(z, \bar{z})}{\partial \bar{z}}, \quad \frac{\partial^{2} P(z, \bar{z})}{\partial z \partial \bar{z}}=0
$$

where the stress functions $P$ and $D$ represent the mean and deviatoric stress, respectively. Using the Kolosov-Muskhelishvili formulae one can present the general solution of plane elasticity as follows:

$$
P(z, \bar{z})=\Phi(z)+\overline{\Phi(z)}, \quad D(z, \bar{z})=\bar{z} \Phi^{\prime}(z)+\Psi(z)
$$

Here $P$ and $D$ are functions of complex variables $z=x-i y, \bar{z}=x-i y$ expressed by two holomorphic functions $\Phi(z), \Psi(z)$ of the complex variable $z$. It is evident from (2) that the stress deviator satisfies the following equation:

$$
\frac{\partial^{2} D(z, \bar{z})}{\partial^{2} \bar{z}}=0
$$


and therefore the elastic problem can be formulated in terms of the stress function $D$ alone. As soon as this function becomes known one can also obtain the mean stress by integration of the equations of equilibrium. This operation introduces an additive constant that cannot be found from the data on orientations of principal stresses.

It should be noted that the deviatoric stress in complex exponential form is:

$$
D(z, \bar{z})=\tau(z, \bar{z}) e^{i \alpha(z, \bar{z})}
$$

where the modulus $\tau(z, \bar{z})$ is associated with the maximum shear stress $\tau_{\max }$ while the argument $\alpha(z, \bar{z})$ is associated with the principal direction $\varphi$ as $\alpha(z, \bar{z})=-2 \varphi(z, \bar{z})$ (in accordance with the geomechanical convention).

The first set of boundary conditions is represented by the argument of the deviatoric stress $(\arg (D(z, \bar{z}))=\alpha(z, \bar{z}))$, which can be expressed in the following form:

$$
\operatorname{Im}\left(e^{-i \alpha^{ \pm}(z, \bar{z})} D^{ \pm}(\zeta, \bar{\zeta})\right)=0, \quad \zeta \in \Gamma
$$

Hereafter the sings \pm distinguish the boundary values for different sides of the domain's boundary.

To complete the formulation of the problem another $\mathrm{BC}$ has to be specified. It assumes continuity of tractions along the margins of the domain:

$$
N^{+}(\zeta)+i T^{+}(\zeta)=N^{-}(\zeta)+i T^{-}(\zeta)
$$

$N, T$ are normal and shear components of the stress vector on $\Gamma$.

The following procedure is further applied for numerical calculations. The complex potentials $\Phi, \Psi$ are sought as polynomials with unknown complex coefficients $c_{k}$ :

$$
\Psi(z)=\sum_{k=0}^{n} c_{k} z^{k}, \quad \Phi(z)=\sum_{k=0}^{n} c_{k+n+1} z^{k}
$$

After substitution of (7) into (2) followed by further substitution of the latter into (5) and (6) one obtains a system of linear algebraic equations with respect to unknown coefficients $c_{k}$ :

$$
\mathbf{A X}=\mathbf{B}
$$

Here it should be noted that this system is not fully homogeneous, it has been complemented by a non-homogeneous equation that assumes that the average value of the maximum shear stress over the entire domain is unity. The system is redundant and therefore it is solved by the least squares method

$$
\mathbf{X}=\left(\mathbf{A}^{T} \mathbf{A}\right)^{-1} \mathbf{A}^{T} \mathbf{B}
$$


The identified coefficients $c_{k}$ are then inserted back to (7) and further to (2) which gives the sought stress functions.

\subsection{Mathematical model in plasticity}

Plastic model is based on classical formulations and numerical approaches developed in $[10,11]$ and complemented by an approach reported recently in Haderka and Galybin [18] that allows extending the solution of the Cauchy boundary value problem beyond the characteristic triangle formed by the slip lines emanating from the boundary. This approach is briefly explained further on.

Based on the Tresca's yield criterion for an ideally plastic material:

$$
\tau=\frac{\sigma_{1}-\sigma_{2}}{2}=\text { const }=c
$$

one can present the Lamé-Maxwell DEE along stress trajectories $s_{1}$ and $s_{2}$ in the following simple form:

$$
\frac{\partial \psi}{\partial s_{1}}+\frac{\partial \varphi}{\partial s_{2}}=0, \quad \frac{\partial \psi}{\partial s_{2}}+\frac{\partial \varphi}{\partial s_{1}}=0
$$

where $\psi=P / 2 c$ and will be referred to as normalised mean stress.

Classical boundary value problem of plasticity assumes that the mean stress $P$ and orientations of principal stresses $\varphi$ can be found by the given stress vector, $N+i T$, on the boundary, and therefore DEE can be easily integrated by the finite difference method. In this study we modified the procedure of numerical integration by using curvilinear net of the stress trajectories instead of the regular grid in Cartesian coordinates that is often used in conventional approaches.

Integration of the equations is performed layer by layer, the first layer being the initial boundary of the problem ( $L$ in Fig. 1$)$. Individual layers are denoted by index $k$ and the position on the layer by $j$.

In order to build the solution along the stress trajectories the Taylor's expansion is used. Accepting the notation in Fig. 1, the orientations and stresses in the layer $z_{j}^{k+1}$ next to the boundary $z_{j}^{k}$, can be written as follows:

$$
\varphi_{j}^{k+1}=\varphi_{j}^{k}+\left.\left|s_{1, j}^{k}\right| \frac{\partial \varphi}{\partial s_{1}}\right|_{j} ^{k} \quad \psi_{j}^{k+1}=\psi_{j}^{k}+\left.\left|s_{1, j}^{k}\right| \frac{\partial \psi}{\partial s_{1}}\right|_{j} ^{k}
$$

The derivatives in (12) are determined from DEE (11). However, they still remain unknown. In order to find them, decomposition to the tangential and normal part is necessary which has the form:

$$
\frac{\partial}{\partial s_{1}}=\cos \gamma \frac{\partial}{\partial t}+\sin \gamma \frac{\partial}{\partial n} \quad \frac{\partial}{\partial s_{2}}=-\sin \gamma \frac{\partial}{\partial t}+\cos \gamma \frac{\partial}{\partial n}
$$

Transformation (13) introduces new unknowns, namely, the normal derivatives of normalised mean stress and of principal orientation which are connected with the contour derivatives as follows: 


$$
\left[\begin{array}{l}
\left.\frac{\partial \psi}{\partial n}\right|_{j} ^{k} \\
\left.\frac{\partial \varphi}{\partial n}\right|_{j} ^{k}
\end{array}\right]=-\left[\begin{array}{cc}
\sin \gamma_{j}^{k} & \cos \gamma_{j}^{k} \\
\cos \gamma_{j}^{k} & \sin \gamma_{j}^{k}
\end{array}\right]^{-1}\left[\begin{array}{cc}
\cos \gamma_{j}^{k} & -\sin \gamma_{j}^{k} \\
-\sin \gamma_{j}^{k} & \cos \gamma_{j}^{k}
\end{array}\right]\left[\begin{array}{l}
\left.\frac{\partial \psi}{\partial t}\right|_{j} ^{k} \\
\left.\frac{\partial \varphi}{\partial t}\right|_{j} ^{k}
\end{array}\right]
$$

Here the tangential derivatives are found from the values at nodes $z_{j}^{k}$. Finally the tangential and normal derivatives may be introduced in (12) to give the orientations and stresses in the nodes $z_{j}^{k+1}$ :

$$
\left[\begin{array}{c}
\varphi_{j}^{k+1} \\
\psi_{j}^{k+1}
\end{array}\right]=\left[\begin{array}{c}
\varphi_{j}^{k} \\
\psi_{j}^{k}
\end{array}\right]+\frac{\left|s_{1, j}^{k}\right|}{\cos 2 \gamma_{j}^{k}}\left[\begin{array}{cc}
\cos \gamma_{j}^{k} & -\sin \gamma_{j}^{k} \\
-\sin \gamma_{j}^{k} & \cos \gamma_{j}^{k}
\end{array}\right]\left[\begin{array}{c}
\left.\frac{\partial \varphi}{\partial t}\right|_{j} ^{k} \\
\left.\frac{\partial \psi}{\partial t}\right|_{j} ^{k}
\end{array}\right]
$$

Equations (15) are solved by employing the forward finite difference scheme. The solution is less stable in the regions where the local angle $\gamma$ is close to $\pm n \pi / 4$, $n=1,2, \ldots$. Nodes $z_{j}^{k+1}$ have to be determined prior to the solution of $\varphi$ and $\psi$ as these coordinates enter the calculations. The basic property of stress trajectories is used here which states that they form an orthogonal grid (different families of stress trajectories intersect at right angles). However, only a linear approximation of stress trajectories is used and tangents, see Fig. 1, to them are used instead the real trajectories (same type of approximation is utilized in classical solution).

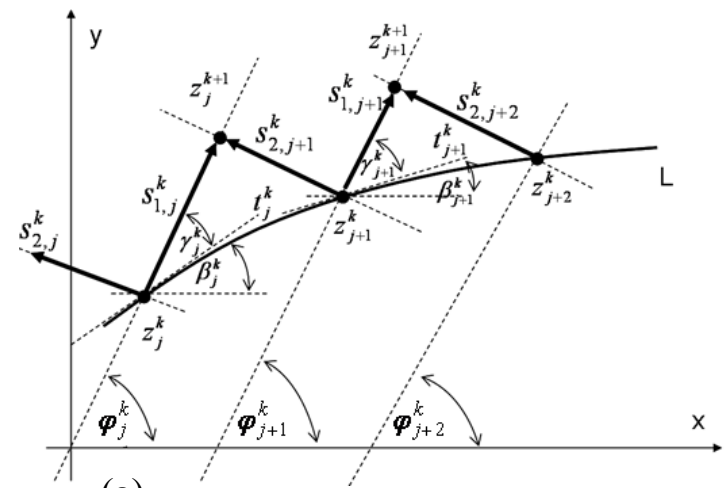

(a)

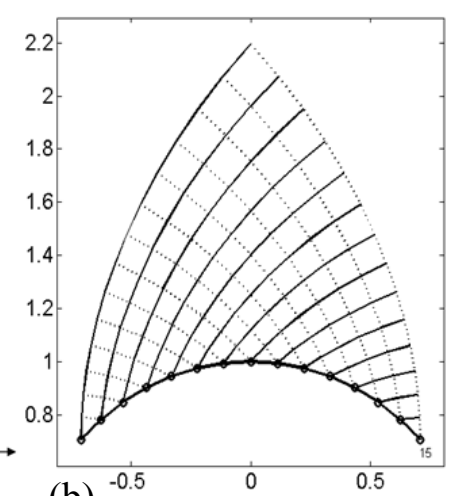

(b)

Figure 1: Determination of the coordinates and visualization of angles (a). Typical solution (characteristic triangle) (b).

\subsection{Plastic model and the alternations of Cauchy's problems}

The idea is based on the relation between lines of slip and stress trajectories which, according to the classical stress theory, are inclined at angles $\pi / 4$ (see Fig. 2(a)). The main reason for the development of the concept of alternations is 
to eliminate the drawback of the classical solutions which is the impossibility to construct solution beyond the initial characteristic triangle (unless some special conditions are imposed, e.g. in Martin [19]).

Let a characteristic triangle be obtained by means of classical methods using the slip lines. Then the principal directions are obtained within this triangle by the rotation of the tangents to the slip lines at the nodes (Fig. 2(b)). Now a new boundary can be formed for the solution along stress trajectories and the numerical scheme, described is subsection 2.3, may be employed. The same procedure can be applied to the solution obtained for the stress trajectories in a different characteristic triangle in order to recover the slip lines.

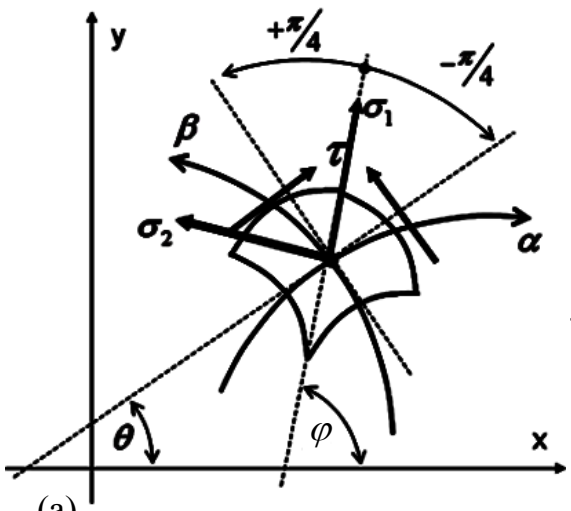

(a)

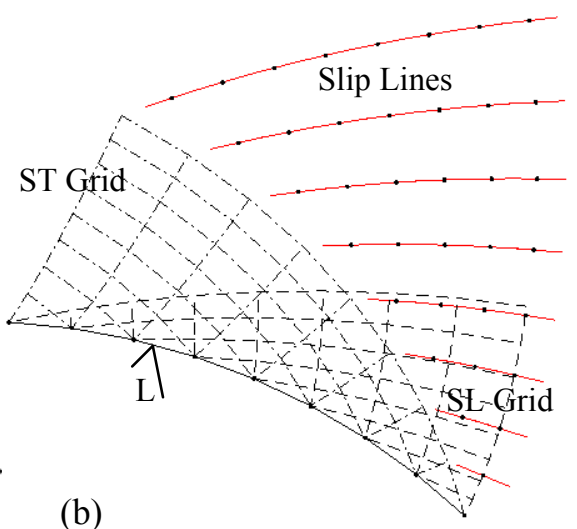

Figure 2: $\quad$ Slip element under normal and shear load (a). Alternations of Cauchy's problems (b).

It is required to modify the $\mathrm{BC}$ properly as the classical methods require the angle of slip as the boundary condition while for building stress trajectories the principal orientations are employed as the BC.

Fig. 2(b) shows a boundary $L$ for which the stress trajectories grid (ST grid) and the slip lines grid (SL grid) have been obtained. It should be mentioned that the results in the area where the triangles intersect correspond perfectly. Moreover the idea of alternations can be noticed from the same picture. The area labelled as Slip Lines is reconstructed by taking the right leg of the ST triangle as a new contour. Note the agreement of the results where the slip lines and SL grid overlaps.

\section{Computational model of Antarctic tectonic plate and results of numerical analysis}

The computational domain for the $2 \mathrm{D}$ elastic problem was built according to Bird's [20] digital model which includes 353 points presenting the margins of the plate. For the purposes of the computations the data which are originally scattered along the margin of the plate had to be separated into internal and external ones. The numerical procedure designed for this objective revealed that 
there are 170 internal data on stress orientations available for the solution. Moreover, data of highest quality were only chosen (quality ranks A-C; according to the WSMP ranking scheme, taken from Reinecker et al [9], these record data to within $\pm 10^{\circ}-25^{\circ}$ precision).

For the plane strain perfectly plastic problem the necessary boundary conditions were taken from the results of the previous elastic analysis (Galybin [12]). The scattered data were approximated along the model's boundary and their number was set to 90 (number of boundary nodal points with mean stress and orientations of principal stress available in them). This allows the comparison of elastic and plastic solutions inside the plate for the same set of boundary conditions.

Fig. 3(a) shows the computational domain, Antarctic tectonic plate, together with the continent of Antarctica and the available data on stress orientations which are clearly scattered along the margins. Fig. 3(b) presents the profiles of the boundary conditions used for plastic analysis (mean stress and orientations of principal stresses).

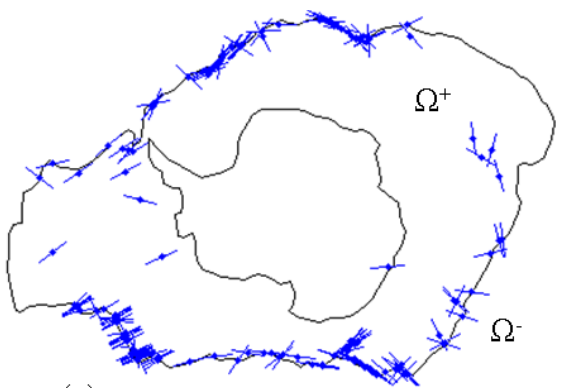

(a)

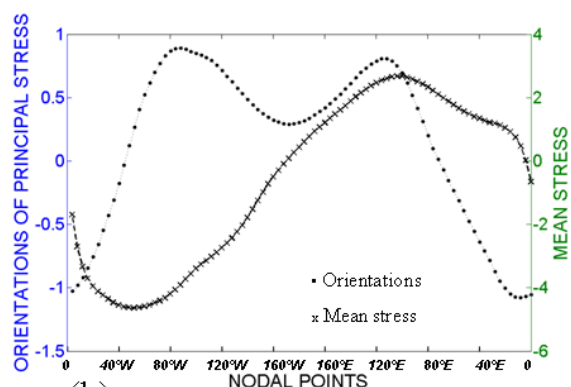

(b)

Figure 3: Antarctic tectonic plate with the data on orientations (a). Smoothened data for plastic solution (b).
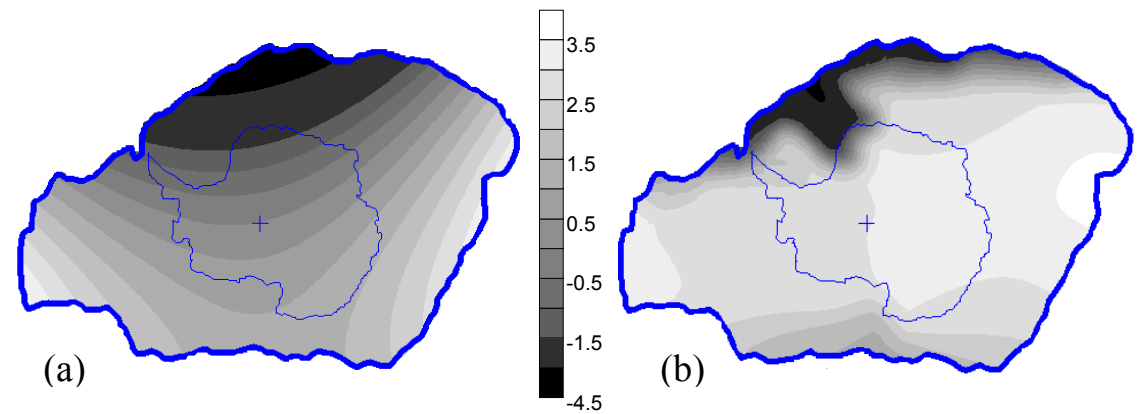

Figure 4: $\quad$ Map of reconstructed stress fields, function $F$, for elastic (a) and plastic (b) rheologies.

For both elastic and plastic problems the maps of mean stresses have been compiled. However, it is necessary to state that only the scaled mean stress 
function $F$ has been recovered which is related to the mean stress as $P=a F+b$ ( $a>0$, and $b$ are free constant parameters of the problem). The maps of this function for both the elastic and plastic cases are shown in Fig. 4.

It is evident from Fig. 4 that the maps are essentially different even for the same boundary values. The reconstructed function $F$ is strongly affected by the type of the rheology selected for the analysis. Thus, in the case of the elastic solution the distribution of mean stresses in the eastern part of the continental lithosphere is more non-uniform as compare to homogeneous one for the plastic case.

\section{Conclusions}

In contrast to other lithospheric plates no attempts have been made so far to determine the stress field in the AP. This is due to the nature of experimental data on stress orientations the majority of which are concentrated at the AP boundaries causing non-applicability of standard approach that needs stress orientations to be known inside a region to constrain solution.

In the present paper two models of the AP stress field have been developed based on the stress orientation concept. They presume either elastic or ideal plastic rheology for the entire AP tectonic plate. It is evident from the results in Fig. 4 that different rheologies lead to essentially different results in modelling tectonic stresses. Therefore the use of purely statistical or interpolation methods, widely applied for studying stresses in the lithosphere, should be avoided and replaced by proper mechanical models that take the rheology into account.

The elastic model is based on numerical solution of the optimisation problem for the data fitting complemented by mechanically justified conditions of continuity of the stress vector across the margins of the adjacent tectonic plates.

The plastic model is investigated by using the concept of alternations of the Cauchy problems for the construction the slip lines and stress trajectories. It is shown that such approach is capable to handle quite complex plane regions, however, verifications regarding convergence and accuracy are still necessary.

\section{Acknowledgement}

The authors acknowledge the support of EPSRC through Research Grant $\mathrm{EP} / \mathrm{E} 032494 / 1$.

\section{References}

[1] Torsvik, T. H., Gaina, C., Redfield, T. F., Antarctica and global paleogeography: From Rodinia, through Gondwanaland and Pangea, to the birth of the Southern Ocean and the opening of Gateways, -In: Cooper A.K. et al., eds. Antarctica: A keystone in a changing world, Proceedings of the 10th International Symposium on Antarctic Earth Sciences, Washington, DC: The National Academies Press, p. 125-140, 2008. 
[2] Gordon, R. G., The Antarctic connection, Nature, 404, p 139-140, 2000.

[3] Sutherland, R., The significance of Antarctica for studies of global geodynamics, -In: Cooper A.K. et al., eds. Antarctica: A keystone in a changing world, Proceedings of the 10th International Symposium on Antarctic Earth Sciences, Washington, DC: The National Academies Press, p. 115-124, 2008.

[4] Cloetingh, S., Wortel, R., Regional stress field of the Indian plate, Geophys. Res. Lett., 12, p. 77-80, 1985.

[5] Coblentz, D. D., Sandiford, M., Richardson, R. M., Zhou S and Hillis, R., The origins of the intraplate stress field in continental Australia. Earth Planet. Sci. Lett., 133, p. 299-309, 1995.

[6] Coblentz, D. D., Sandiford, M., Tectonic stresses in the African plate: Constraints on the ambient lithospheric stress state, Geology, 22, p. 831834, 1994.

[7] Gölke, M., Coblenz, D., Origins of the European regional stress field, Tectonophysics, 266, p. 11-24, 1996.

[8] Humphreys, E. D., Coblentz, D. D., North American dynamics and western U.S. tectonics, Rev. Geophys, v. 45, RG3001, doi:10.1029/2005RG000181, 2007.

[9] Reinecker, J., Heidbach, O., Tingay, M., Sperner, B., Müller, B., The release 2005 of the World Stress Map // 2005, available online at www.world-stress-map.org.

[10] Hill, R., The Mathematical Theory of Plasticity, Oxford: Clarendon Press, 1950.

[11] Kachanov, L. M., Fundamentals of Theory of Plasticity, Published: Foundations of the theory of plasticity, Amsterdam, 1971.

[12] Galybin, A. N., Stress fields in joined elastic regions: Modelling based on discrete stress orientations, In Situ Rock Stress - Lu, Li, Kjerholt \& Dahle (eds), Taylor \& Francis/Balkema, Leiden, p. 193-199, 2006.

[13] Sykes, L., Intraplate seismicity, reactivation of pre-existing zone of weakness, alkaline magmatism and other tectonism postdating continental fragmentation, J. Geophys. Res., 16 (B), p. 621-687, 1978.

[14] Okal, E. A., The Bellingshausen sea earthquake of February 5, 1977: Evidence for ridge-generated compression in the Antarctic plate, Earth Planet. Sci. Lett., 46, p. 306-310, 1980.

[15] Kreemer, C., Holt, W. E., What Caused the March 25, 1998 Antarctic Plate Earthquake?: Inferences from Regional Stress and Strain Rate Fields, Geophys. Res. Lett., 27, p. 2297-2300, 2000.

[16] Mukhamediev, Sh. A., Galybin, A. N., Brady, B. H. G., Determination of stress fields in the elastic lithosphere by methods based on stress orientations, Int. J. of Rock Mech. \& Mining Sci., 43, p. 66-88, doi:10.1016/j.ijrmms.2005.04.008, 2006.

[17] Galybin, A. N., Mukhamediev Sh. A., Determination of Elastic Stresses from Discrete Data on Stress Orientations, Int. J. of Sol. and Struc., 41, 5125-5142, 2004. 
[18] Haderka P., Galybin A. N., Damage zones near excavations: Plastic solution by means of stress trajectories, First international conference on underground spaces - design, engineering and environmental aspects, 1, 109-119, 2008.

[19] Martin, C. M., Exact bearing capacity calculations using the method of characteristics, Proc. 11th Int. Conf. of IACMAG, Turin, 4, pp 441-450, 2005

[20] Bird, P., An updated digital model for plate boundaries, Geochem. Geophys. Geosyst., 4(3), 1027, doi: 10.1029/2001GC000252, 2003. 